\title{
Application of the Generalized Integral Representation Method (GIRM) to Tidal Wave Propagation
}

\author{
Hiroshi Isshiki \\ IMA, Institute of Mathematical Analysis, Osaka, Japan
}

Email address:

isshiki@dab.hi-ho.ne.jp

To cite this article:

Hiroshi Isshiki. Application of the Generalized Integral Representation Method (GIRM) to Tidal Wave Propagation. Applied and Computational Mathematics. Special Issue: Integral Representation Method and its. Vol. 4, No. 3-1, 2015, pp. 52-58.

doi: 10.11648/j.acm.s.2015040301.14

\begin{abstract}
Integral Representation Method (IRM) is one of convenient methods to solve Initial and Boundary Value Problems (IBVP). It can be applied to irregular mesh, and the solution is stable and accurate. IRM is developed to Generalized Integral Representation Method (GIRM) to treat any kinds of problems including nonlinear problems. In GIRM, Generalized Fundamental Solution (GFS) is used instead of Fundamental Solution (FS) in IRM. We can use a variety of GFSs in GIRM. The effects of typical GFSs are investigated. In the present paper, an application of GIRM to tidal wave propagation is discussed, and the time evolution involves the second order time derivatives. An explicit time evolution is used successfully in the present paper.
\end{abstract}

Keywords: Initial and Boundary Value Problems (IBVP), Generalized Integral Representation Method (GIRM), Generalized Fundamental Solution (GFS), Second Order Time Derivatives in Time Evolution, Tidal Wave Propagation

\section{Introduction}

Integral Representation Method (IRM) [1-3] is one of convenient methods to solve Initial and Boundary Value Problems (IBVP). It can be applied to irregular mesh, and the solution is stable and accurate. IRM was originally developed to solve linear boundary value problems, and the Fundamental Solution (FS) of the original differential equation plays a major role in IRM. IRM is developed to Generalized Integral Representation Method (GIRM) [4-8] to treat any kinds of problems including nonlinear problems. GIRM uses Generalized Fundamental Solution (GFS). GFS is not required to satisfy the differential equation of the original problem. We can use various GFSs for an individual problem. It is very important to know the characteristics of each GFS.

In the present paper, an application of GIRM to tidal wave propagation is discussed. In this problem, the time evolution involves the second order time derivatives.

\section{Initial and Boundary Value Problem (IBVP)}

\subsection{Shallow Water Wave Problem}

In the present paper, a very simple tidal wave problem in horizontal space $S$ with boundary $C$ is discussed:

$$
\begin{gathered}
\frac{\partial^{2} \eta(\mathbf{x}, t)}{\partial t^{2}}=\nabla \cdot(g h(\mathbf{x}) \nabla \eta(\mathbf{x}, t)) \text { in } S \text { for } t>0, \\
\frac{\partial \eta(\mathbf{x}, t)}{\partial n}=0 \text { on } C \text { for } t>0, \\
\eta(\mathbf{x}, t)=f(\mathbf{x}), \frac{\partial \eta(\mathbf{x}, t)}{\partial t}=g(\mathbf{x}) \text { on } C \text { at } t=0,
\end{gathered}
$$


where $\mathbf{x}=(x, y)$ and $t$ are horizontal coordinates and time, respectively. $\nabla$ and $\partial / \partial n$ are operator nabla $(\partial / \partial x, \partial / \partial y)$ and outward normal derivative on $C$, respectively. $\eta(\mathbf{x}, t)$, $h(\mathbf{x})$ and $g$ are surface elevation, water depth and gravitational acceleration, respectively. $f(\mathbf{x})$ and $g(\mathbf{x})$ are given functions.

\subsection{1-Step Generalized Integral Representation Method (1-Step GIRM)}

Let $\tilde{G}(\mathbf{x}, \boldsymbol{\xi})$ be a Generalized Fundamental Solution (GFS) such as $[7,8]$

$$
\tilde{\eta}(\mathbf{x}, \xi, t)=\tilde{\eta}(\mathbf{x}, \xi)=\frac{1}{2 \pi \gamma^{2}} \exp \left(-\frac{|\mathbf{x}-\xi|^{2}}{2 \gamma^{2}}\right)
$$

From Eq. (1), we have

$$
\begin{aligned}
0 & =\iint_{S_{\mathbf{x}}}\left[\frac{\partial^{2} \eta(\mathbf{x}, t)}{\partial t^{2}}-\nabla_{\mathbf{x}} \cdot\left(g h(\mathbf{x}) \nabla_{\mathbf{x}} \eta(\mathbf{x}, t)\right)\right] \tilde{G}(\mathbf{x}, \xi) d S_{\mathbf{x}} \\
& =\iint_{S_{\mathbf{x}}} \frac{\partial^{2} \eta(\mathbf{x}, t)}{\partial t^{2}} \tilde{G}(\mathbf{x}, \xi) d S_{\mathbf{x}} \\
& -\iint_{S_{\mathbf{x}}} \nabla_{\mathbf{x}} \cdot\left(g h(\mathbf{x}) \tilde{G}(\mathbf{x}, \xi) \nabla_{\mathbf{x}} \eta(\mathbf{x}, t)\right) d S_{\mathbf{x}} \\
& +\iint_{S_{\mathbf{x}}}\left(g h(\mathbf{x}) \nabla_{\mathbf{x}} \eta(\mathbf{x}, t)\right) \cdot \nabla_{\mathbf{x}} \tilde{G}(\mathbf{x}, \xi) d S_{\mathbf{x}} \\
& =\iint_{S_{\mathbf{x}}} \frac{\partial^{2} \eta(\mathbf{x}, t)}{\partial t^{2}} \tilde{G}(\mathbf{x}, \xi) d S_{\mathbf{x}} \\
& -\iint_{S_{\mathbf{x}}} \nabla_{\mathbf{x}} \cdot\left(g h(\mathbf{x}) \tilde{G}(\mathbf{x}, \xi) \nabla_{\mathbf{x}} \eta(\mathbf{x}, t)\right) d S_{\mathbf{x}} \\
& +\iint_{S_{\mathbf{x}}} \nabla_{\mathbf{x}} \cdot\left[\eta(\mathbf{x}, t) g h(\mathbf{x}) \nabla_{\mathbf{x}} \tilde{G}(\mathbf{x}, \xi)\right] d S_{\mathbf{x}} \\
& -\iint_{S_{\mathbf{x}}} \eta(\mathbf{x}, t) \nabla_{\mathbf{x}} \cdot\left(g h(\mathbf{x}) \nabla_{\mathbf{x}} \tilde{G}(\mathbf{x}, \xi)\right) d S_{\mathbf{x}}
\end{aligned}
$$

Rewriting Eq. (5), we obtain

$$
\begin{aligned}
& \iint_{S_{\mathbf{x}}} \frac{\partial^{2} \eta(\mathbf{x}, t)}{\partial t^{2}} \tilde{G}(\mathbf{x}, \xi) d S_{\mathbf{x}} \\
& =\iint_{S_{\mathbf{x}}} \eta(\mathbf{x}, t) \nabla_{\mathbf{x}} \cdot\left(g h(\mathbf{x}) \nabla_{\mathbf{x}} \tilde{G}(\mathbf{x}, \xi)\right) d S_{\mathbf{x}} \\
& +\int_{C_{\mathbf{x}}} g h(\mathbf{x}) \tilde{G}(\mathbf{x}, \xi) \frac{\partial \eta(\mathbf{x}, t)}{\partial n_{\mathbf{x}}} d C_{\mathbf{x}} \\
& -\int_{C_{\mathbf{x}}} g h(\mathbf{x}) \eta(\mathbf{x}, t) \frac{\partial \tilde{G}(\mathbf{x}, \xi)}{\partial n_{\mathbf{x}}} d C_{\mathbf{x}}
\end{aligned}
$$

Exchanging $\mathbf{x}$ and $\xi$, we derive the General Integral Representation (GIR) of Eqs. (1)

$$
\begin{aligned}
& \iint_{S_{\xi}} \frac{\partial^{2} \eta(\xi, t)}{\partial t^{2}} \tilde{G}(\boldsymbol{\xi}, \mathbf{x}) d S_{\xi} \\
& =\iint_{S_{\xi}}\left[\eta(\xi, t) \nabla_{\xi} \cdot\left(g h(\xi) \nabla_{\xi} \tilde{G}(\boldsymbol{\xi}, \mathbf{x})\right)\right] d S_{\xi} \\
& +\int_{C_{\xi}} g h(\xi) \tilde{G}(\xi, \mathbf{x}) \frac{\partial \eta(\xi, t)}{\partial n_{\xi}} d C_{\xi} \\
& -\int_{C_{\xi}} g h(\xi) \eta(\xi, t) \frac{\partial \tilde{G}(\boldsymbol{\xi}, \mathbf{x})}{\partial n_{\xi}} d C_{\xi}
\end{aligned}
$$

Let $\delta(x)$ be Dirac's delta function. If $\nabla_{\xi} \tilde{G}(\xi, \mathbf{x})$ satisfies

$$
\nabla_{\xi} \cdot\left(g h(\xi) \nabla_{\xi} \tilde{G}(\xi, \mathbf{x})\right)=\delta(\xi-x) \delta(\eta-y),
$$

the first integral on the right-hand side of Eq. (7) becomes $\eta(\mathbf{x}, t)$. Hence, Eq. (7) becomes an integral representation of $\eta(\mathbf{x}, t)$.

If $\eta(x, t)$ at internal point (IP) is known and $\eta(x, t)$ or $\partial \eta(x, t) / \partial n$ at boundary point (BP) is known from the boundary conditions, then the generalized integral representations are integral equations with $\partial^{2} \eta(x, t) / \partial t^{2}$ the unknown variables at IP and $\partial \eta(x, t) / \partial n$ or $\eta(x, t)$ the unknown variables at BP. We obtain $\eta(x, t+d t)$ at IP from $\partial \eta(x, t+d t) / \partial t=\partial \eta(x, t) / \partial t+d t \partial^{2} \eta(x, t) / \partial t^{2} \quad$ and $\eta(x, t+d t)=\eta(x, t)+d t \partial \eta(x, t+d t) / \partial t$. If $\eta(x, 0)$ and $\partial \eta(x, 0) / \partial t$ on IP are known from the initial conditions, then we can solve IBVP using the GIR. The GIRs are equivalent to the differential equations.

\subsection{2-Step Generalized Integral Representation Method (2-Step GIRM)}

First, we rewrite Eq. (1) as follows.

(a) Non-uniformity equation:

$$
\boldsymbol{\theta}(\mathbf{x}, t)=\nabla \eta(\mathbf{x}, t) .
$$

(b) Constitutive equation:

$$
\mathbf{q}(\mathbf{x}, t)=\operatorname{gh}(\mathbf{x}) \boldsymbol{\theta}(\mathbf{x}, t) .
$$

(c) Equilibrium equation:

$$
\frac{\partial^{2} \eta(\mathbf{x}, t)}{\partial t^{2}}=\nabla \cdot \mathbf{q}(\mathbf{x}, t)
$$

From Equations (9), we have

$$
\begin{aligned}
0 & =\iint_{S_{\mathbf{x}}}\left[\boldsymbol{\theta}(\mathbf{x}, t)-\nabla_{\mathbf{x}} \eta(\mathbf{x}, t)\right] \tilde{G}(\mathbf{x}, \xi) d S_{\mathbf{x}} \\
& =\iint_{S_{\mathbf{x}}} \tilde{G}(\mathbf{x}, \xi) \boldsymbol{\theta}(\mathbf{x}, t) d S_{\mathbf{x}} \\
& -\iint_{S_{\mathbf{x}}}\left[\nabla_{\mathbf{x}}(\tilde{G}(\mathbf{x}, \boldsymbol{\xi}) \eta(\mathbf{x}, t))-\eta(\mathbf{x}, t) \nabla_{\mathbf{x}} \tilde{G}(\mathbf{x}, \xi)\right] d S_{\mathbf{x}}
\end{aligned}
$$

Rewriting Eq. (12), we have

$$
\begin{aligned}
\iint_{S_{\mathbf{x}}} \tilde{G}(\mathbf{x}, \xi) \boldsymbol{\theta}(\mathbf{x}, t) d S_{\mathbf{x}} & =-\iint_{S_{\mathbf{x}}}\left[\eta(\mathbf{x}, t) \nabla_{\mathbf{x}} \tilde{G}(\mathbf{x}, \xi)\right] d S_{\mathbf{x}} \\
& +\int_{C_{\mathbf{x}}} \tilde{G}(\mathbf{x}, \xi) \eta(\mathbf{x}, t) \mathbf{n}_{\mathbf{x}} d C_{\mathbf{x}}
\end{aligned}
$$

where $\mathbf{n}$ is the unit outward normal vector of the boundary $C$. Exchanging $\mathbf{x}$ and $\xi$, we obtain

$$
\begin{aligned}
\iint_{S_{\xi}} \tilde{G}(\boldsymbol{\xi}, \mathbf{x}) \boldsymbol{\theta}(\boldsymbol{\xi}, t) d S_{\xi} & =-\iint_{S_{\xi}}\left[\eta(\xi, t) \nabla_{\xi} \tilde{G}(\boldsymbol{\xi}, \mathbf{x})\right] d S_{\xi} \\
& +\int_{C_{\xi}} \tilde{G}(\boldsymbol{\xi}, \mathbf{x}) \eta(\xi, t) \mathbf{n}_{\xi} d C_{\xi}
\end{aligned}
$$


From Eq. (11), we have

$$
\begin{aligned}
0 & =\iint_{S_{\mathbf{x}}}\left[\frac{\partial^{2} \eta(\mathbf{x}, t)}{\partial t^{2}}-\nabla_{\mathbf{x}} \cdot \mathbf{q}(\mathbf{x}, t)\right] \tilde{G}(\mathbf{x}, \xi) d S_{\mathbf{x}} \\
& =\iint_{S_{\mathbf{x}}} \tilde{G}(\mathbf{x}, \xi) \frac{\partial^{2} \eta(\mathbf{x}, t)}{\partial t^{2}} d S_{\mathbf{x}} \\
& -\iint_{S_{\mathbf{x}}} \nabla_{\mathbf{x}} \cdot(\tilde{G}(\mathbf{x}, \xi) \mathbf{q}(\mathbf{x}, t)) d S_{\mathbf{x}}+\iint_{S_{\mathbf{x}}} \nabla_{\mathbf{x}} \tilde{G}(\mathbf{x}, \xi) \cdot \mathbf{q}(\mathbf{x}, t) d S_{\mathbf{x}}
\end{aligned}
$$

Rewriting Eq. (15), we obtain

$$
\begin{aligned}
& \iint_{S_{\mathbf{x}}} \tilde{G}(\mathbf{x}, \boldsymbol{\xi}) \frac{\partial^{2} \eta(\mathbf{x}, t)}{\partial t^{2}} d S_{\mathbf{x}} \\
& =-\iint_{S_{\mathbf{x}}} \nabla_{\mathbf{x}} \tilde{G}(\mathbf{x}, \boldsymbol{\xi}) \cdot \mathbf{q}(\mathbf{x}, t) d S_{\mathbf{x}}+\int_{C_{\mathbf{x}}} \tilde{G}(\mathbf{x}, \boldsymbol{\xi}) \mathbf{q}(\mathbf{x}, t) \cdot \mathbf{n}_{\mathbf{x}} d C_{\mathbf{x}}
\end{aligned}
$$

Exchanging $\mathbf{x}$ and $\xi$ in Eq. (16), we derive

$$
\begin{aligned}
& \iint_{S_{\xi}} \tilde{G}(\xi, \mathbf{x}) \frac{\partial^{2} \eta(\xi, t)}{\partial t^{2}} d S_{\xi} \\
& =-\iint_{S_{\xi}} \nabla_{\xi} \tilde{G}(\xi, \mathbf{x}) \cdot \mathbf{q}(\xi, t) d S_{\xi}+\int_{C_{\xi}} \tilde{G}(\xi, \mathbf{x}) \mathbf{q}(\xi, t) \cdot \mathbf{n}_{\xi} d C_{\xi}
\end{aligned}
$$

Equations (14) and (17) are GIRs of Eqs. (9) and (11), respectively.

If $\eta(\mathbf{x}, t)$ at IP is known, then GIR given by Eq. (14) is an integral equation with $\boldsymbol{\theta}(\mathbf{x}, t)$ the unknown variable at IP and $\eta(\mathbf{x}, t)$ the unknown variables at BP. These unknown variables are obtained solving the integral equation. $\mathbf{q}(\mathbf{x}, t)$ at IP is obtained from Equation (10). Then, GIR given by Eq. (17) is an integral equation with $\partial^{2} \eta(\mathbf{x}, t) / \partial t^{2}$ the unknown variable at IP and $\mathbf{q}(\mathbf{x}, t)$ the unknown variables at BP. These unknown variables are obtained solving the integral equation. We obtain $\partial \eta(\mathbf{x}, t+d t) / \partial t$ and $\eta(\mathbf{x}, t+d t)$ at IP from $\partial \eta(\mathbf{x}, t+d t) / \partial t=\partial \eta(\mathbf{x}, t) / \partial t+d t \partial^{2} \eta(\mathbf{x}, t) / \partial t^{2} \quad$ and $\eta(\mathbf{x}, t+d t)=\eta(\mathbf{x}, t)+d t \partial \eta(\mathbf{x}, t+d t) / \partial t$, respectively. We repeat the procedure mentioned above at every time step. If $\eta(x, 0)$ and $\partial \eta(x, 0) / \partial t$ on IP are known from the initial conditions, then we can solve IBVP using GIRs. GIRs are equivalent to the differential equations.

\section{Numerical Results}

For simplicity, we focus on one-dimensional problem in region $-L<x<+L$ given by

$$
\begin{gathered}
\frac{\partial^{2} \eta(x, t)}{\partial t^{2}}=\frac{\partial}{\partial x}\left(g h(x) \frac{\partial \eta(x, t)}{\partial x}\right), \\
\text { in }-L<x<+L \text { for } t>0 \\
\frac{\partial \eta(+L, t)}{\partial x}=0, \quad \frac{\partial \eta(-L, t)}{\partial x}=0 \text { for } t>0, \\
\eta(x, 0)=f(x), \frac{\partial \eta(x, 0)}{\partial t}=g(x) . \\
\text { in }-L<x<+L
\end{gathered}
$$

We use four kinds of GFSs [8]:

(1) $C^{0}$ Continuous $\mathbf{G F S}$

Harmonic GFS

$$
\begin{gathered}
\tilde{G}(x, \xi)=\frac{1}{2}|x-\xi|, \\
\frac{\partial \tilde{G}(x, \xi)}{\partial x}=\frac{1}{2} \operatorname{sgn}(x-\xi), \\
\frac{\partial^{2} \tilde{G}(x, \xi)}{\partial x^{2}}=\delta(x-\xi),
\end{gathered}
$$

Exponential GFS

$$
\begin{gathered}
\tilde{G}(x, \xi)=-\frac{\gamma}{2} \exp \left(-\frac{|x-\xi|}{\gamma}\right), \\
\frac{\partial \tilde{G}(x, \xi)}{\partial x}=\frac{1}{2} \exp \left(-\frac{|x-\xi|}{\gamma}\right) \operatorname{sgn}(x-\xi), \\
\frac{\partial^{2} \tilde{G}(x, \xi)}{\partial x^{2}}=-\frac{1}{2 \gamma} \exp \left(-\frac{|x-\xi|}{\gamma}\right)+\delta(x-\xi) .
\end{gathered}
$$

(2) $C^{2}$ Continuous GFS [8-10]

Lucy GFS

$$
\begin{aligned}
& \tilde{G}(x, \xi)=\left\{\begin{array}{c}
\frac{5}{4} \frac{1}{\gamma}\left(1+3 \frac{|x-\xi|}{\gamma}\right)\left(1-\frac{|x-\xi|}{\gamma}\right)^{3},|x-\xi| \leq \gamma \\
0, \quad|x-\xi|>\gamma
\end{array},\right. \\
& \frac{\partial \tilde{G}(x, \xi)}{\partial x}=\left\{\begin{array}{c}
{\left[\frac{5}{4} \frac{3}{\gamma^{2}}\left(1-\frac{|x-\xi|}{\gamma}\right)^{3}-\frac{5}{4} \frac{3}{\gamma^{2}}\left(1+3 \frac{|x-\xi|}{\gamma}\right)\left(1-\frac{|x-\xi|}{\gamma}\right)^{2}\right] .} \\
\cdot \operatorname{sgn}(x-\xi),|x-\xi| \leq \gamma \\
0, \quad|x-\xi|>\gamma
\end{array},\right. \\
& \frac{\partial^{2} \tilde{G}(x, \xi)}{\partial x^{2}}=\left\{\begin{array}{c}
{\left[\begin{array}{c}
\left.-\frac{5}{4} \frac{9}{\gamma^{3}}\left(1-\frac{|x-\xi|}{\gamma}\right)^{2}+\frac{5}{4} \frac{6}{\gamma^{3}}\left(1+3 \frac{|x-\xi|}{\gamma}\right)\left(1-\frac{|x-\xi|}{\gamma}\right)\right], \\
|x-\xi| \leq \gamma
\end{array}\right.} \\
0, \quad|x-\xi|>\gamma
\end{array} .\right.
\end{aligned}
$$

(3) $C^{\infty}$ Continuous GFS

Gaussian GFS

$$
\begin{gathered}
\tilde{G}(x, \xi)=\frac{1}{\sqrt{2 \pi} \gamma} \exp \left(-\frac{(x-\xi)^{2}}{2 \gamma^{2}}\right) \\
\frac{\partial \tilde{G}(x, \xi)}{\partial x}=-\frac{1}{\sqrt{2 \pi} \gamma^{3}}(x-\xi) \exp \left(-\frac{(x-\xi)^{2}}{2 \gamma^{2}}\right) \\
\frac{\partial^{2} \tilde{G}(x, \xi)}{\partial x^{2}}=-\frac{1}{\sqrt{2 \pi} \gamma^{3}} \exp \left(-\frac{(x-\xi)^{2}}{2 \gamma^{2}}\right) \\
+\frac{1}{\sqrt{2 \pi} \gamma^{5}}(x-\xi)^{2} \exp \left(-\frac{(x-\xi)^{2}}{2 \gamma^{2}}\right)
\end{gathered}
$$




\subsection{Travelling of a Wave in Infinite One-Dimensional Space}

For this problem, we use One-Step GIRM. We rewrite Eq. (7) for one-dimensional case:

$$
\begin{aligned}
& \int_{-L}^{+L} \frac{\partial^{2} \eta(\xi, t)}{\partial t^{2}} \tilde{G}(\xi, x) d \xi=\int_{-L}^{+L} \eta(\xi, t) \frac{\partial}{\partial \xi}\left(g h(\xi) \frac{\partial \tilde{G}(\xi, x)}{\partial \xi}\right) d \xi \\
& +\left[g h(+L) \tilde{G}(+L, x) \frac{\partial \eta(+L, t)}{\partial \xi}-g h(-L) \tilde{G}(-L, x) \frac{\partial \eta(-L, t)}{\partial \xi}\right] . \\
& -\left[g h(+L) \eta(+L, t) \frac{\partial \tilde{G}(+L, x)}{\partial \xi}-g h(-L) \eta(-L, t) \frac{\partial \tilde{G}(-L, x)}{\partial \xi}\right]
\end{aligned}
$$

If we consider infinite one-dimensional space, $L$ is taken large enough, and we assume

$$
\frac{\partial C( \pm L, t)}{\partial x}=0 \text { and } C( \pm L, t)=0 .
$$

Equation (25) is now approximated by

$$
\int_{-L}^{+L} \frac{\partial^{2} \eta(\xi, t)}{\partial t^{2}} \tilde{G}(\xi, x) d \xi=\int_{-L}^{+L} \eta(\xi, t) \frac{\partial}{\partial \xi}\left(g h(\xi) \frac{\partial \tilde{G}(\xi, x)}{\partial \xi}\right) d \xi .
$$

The time evolution with time step $d t$ is given by

$$
\begin{gathered}
\frac{\partial \eta(x, t+d t)}{\partial t} \approx \frac{\partial \eta(x, t)}{\partial t}+\frac{\partial^{2} \eta(x, t)}{\partial t^{2}} d t \\
\eta(x, t+d t) \approx \eta(x, t)+\frac{\partial \eta(x, t+d t)}{\partial t} d t
\end{gathered}
$$

The initial value is specified as

$$
\eta(x, 0)=\exp \left(-\frac{x^{2}}{2(L / 8)^{2}}\right),-L<x<L .
$$

The discretization of Eq. (27) is briefly discussed in Appendix A [7,8]. The solution of the Initial Boundary Value Problem (IBVP) is summarized as

$$
\text { From Eq. (27), } \eta(\mathbf{x}, t) \rightarrow \partial^{2} \eta(\mathbf{x}, t) / \partial t^{2}
$$$$
\text { from Eq. (28a), } \partial^{2} \eta(\mathbf{x}, t) / \partial t^{2} \rightarrow \partial \eta(\mathbf{x}, t+d t) / \partial t ;
$$$$
\text { from Eq. (28b), } \partial \eta(\mathbf{x}, t+d t) / \partial t \rightarrow \eta(\mathbf{x}, t+d t) \text {; }
$$

repeat.

Parameters for numerical calculations are given below:

$$
\begin{gathered}
c=\sqrt{g h}=2 ; L=4 ; N=80 ; \\
d x=2 L / N=0.1 ; d t=0.001,
\end{gathered}
$$

where $N$ is the number of elements in the region $-L<x<L$.

The exact solution is given in Fig. 1, and the numerical results are given in Fig. 2. The accurate results are obtained among numerical results using Harmonic, Exponential and
Gaussian GFSs. The numerical result using Lucy GFS is not accurate enough.

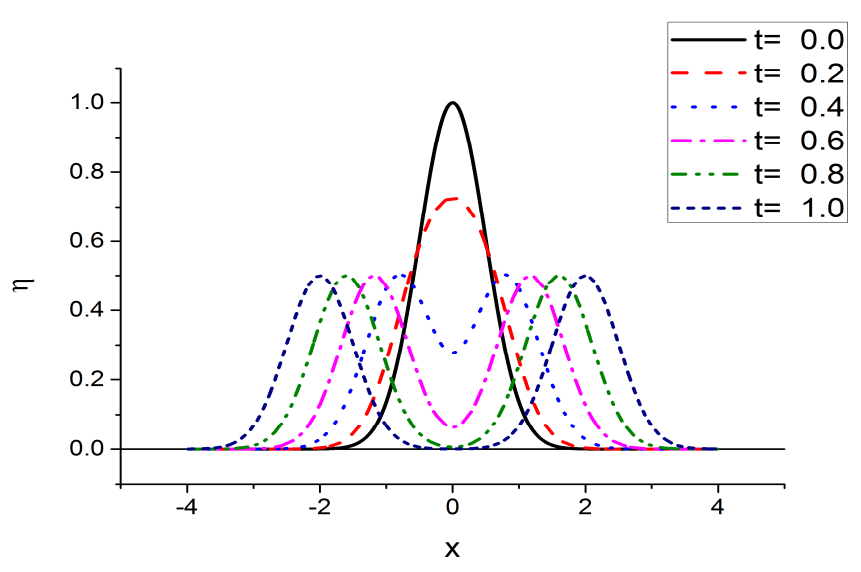

Figure 1. Exact solution of $\eta(x, t)$

\subsection{Reflection of a Wave by Walls at Boundaries}

For this problem, we use Two-Step GIRM. We rewrite Eqs. (14) and (17) for one-dimensional case:

$$
\begin{aligned}
& \int_{-L}^{+L} \tilde{G}(\xi, x) \theta(\xi, t) d \xi \\
& =-\int_{-L}^{+L} \eta(\xi, t) \frac{\partial \tilde{G}(\xi, x)}{\partial \xi} d \xi \\
& +[\tilde{G}(+L, x) \eta(+L, t)-\tilde{G}(-L, x) \eta(-L, t)] \\
& \int_{-L}^{+L} \tilde{G}(\xi, x) \frac{\partial^{2} \eta(\xi, t)}{\partial t^{2}} d \xi \\
& =-\int_{-L}^{+L} \frac{\partial \tilde{G}(\xi, x)}{\partial \xi} q(\xi, t) d \xi \\
& +[\tilde{G}(+L, x) q(+L, t)-\tilde{G}(-L, x) q(-L, t)]
\end{aligned}
$$

The boundary values $\eta(+L, t)$ and $\eta(-L, t)$ are approximated, for example, by

$$
\eta(+L, t) \approx \eta\left(x_{N-1}, t\right), \eta(-L, t) \approx \eta\left(x_{0}, t\right),
$$

where $x_{i}$ is given by $x_{i}=-L+(i+0.5) L / N$, $i=0,1, \cdots, N-1$. Then, Eq. (32) rewritten as

$$
\begin{aligned}
& \int_{-L}^{+L} \tilde{G}(\xi, x) \theta(\xi, t) d \xi \\
& =-\int_{-L}^{+L} \eta(\xi, t) \frac{\partial \tilde{G}(\xi, x)}{\partial \xi} d \xi \\
& +\left[\tilde{G}(+L, x) \eta\left(x_{N-1}, t\right)-\tilde{G}(-L, x) \eta\left(x_{0}, t\right)\right]
\end{aligned}
$$

And substituting the boundary condition (19), Eq. (33) becomes

$$
\int_{-L}^{+L} \tilde{G}(\xi, x) \frac{\partial^{2} \eta(\xi, t)}{\partial t^{2}} d \xi=-\int_{-L}^{+L} \frac{\partial \tilde{G}(\xi, x)}{\partial \xi} q(\xi, t) d \xi .
$$


The same time evolution (28) and initial condition (29) are used.

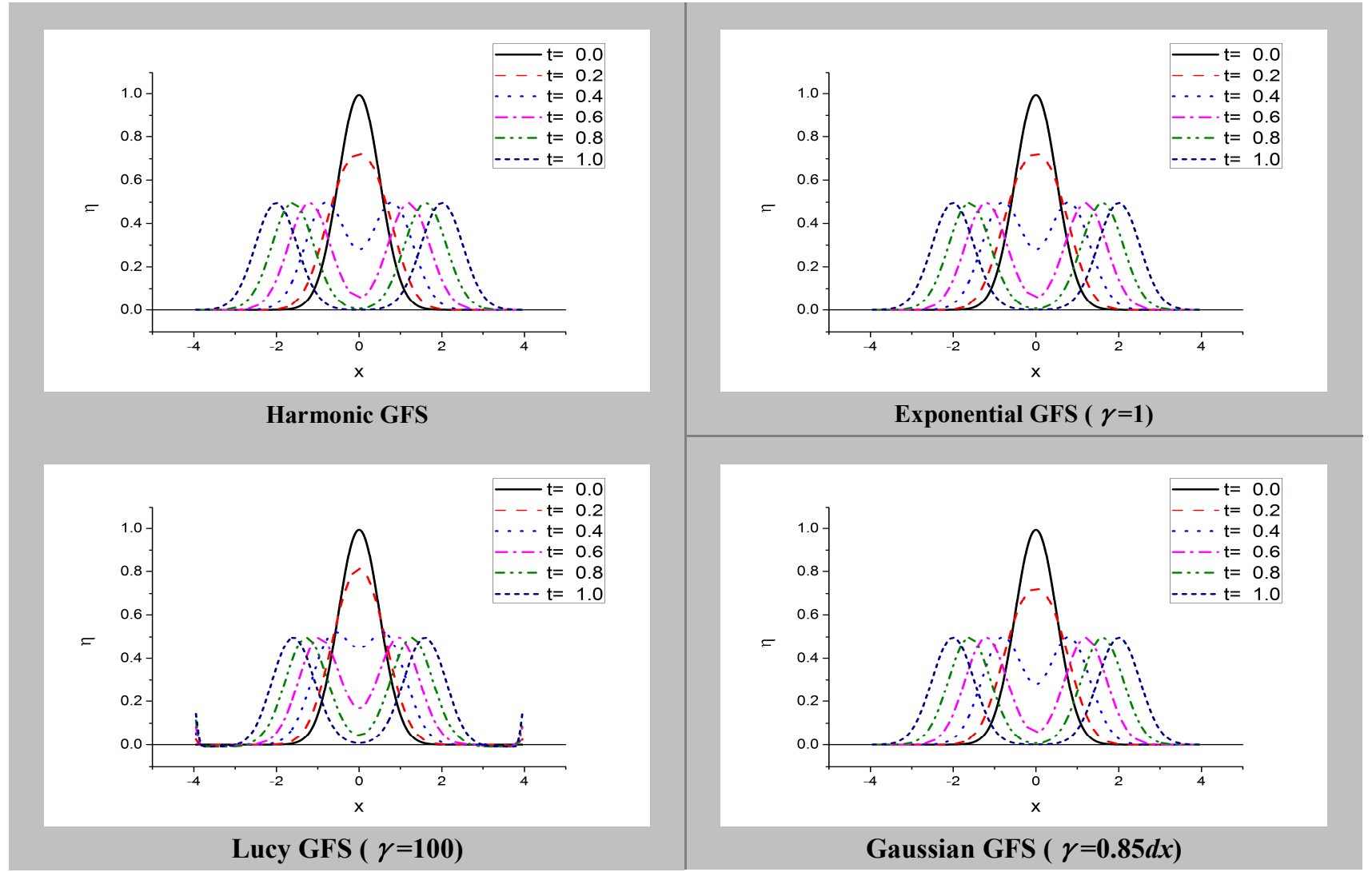

Figure 2. Numerical results using various GFSs

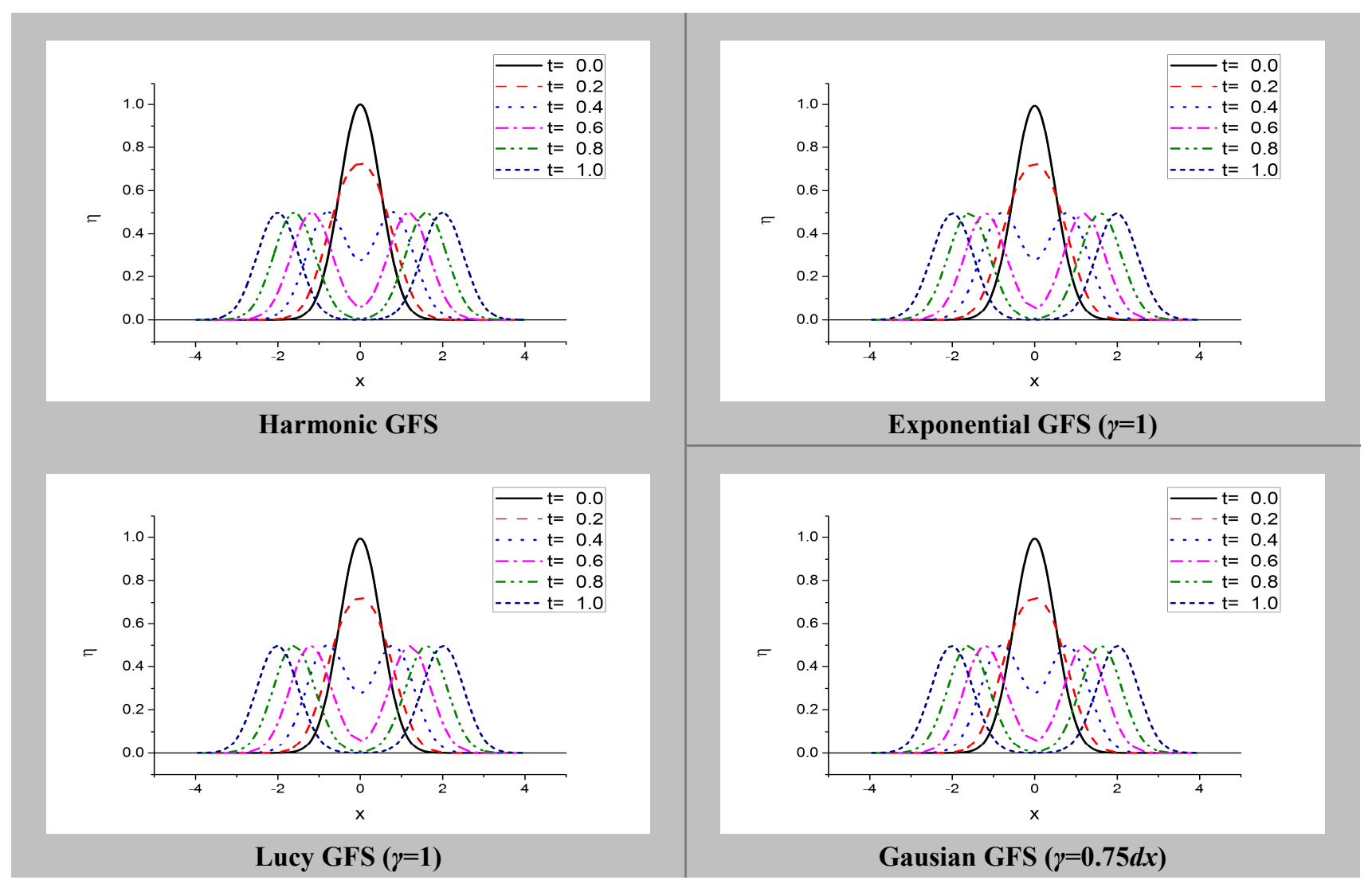

Figure 3. Without reflection at boundaries 


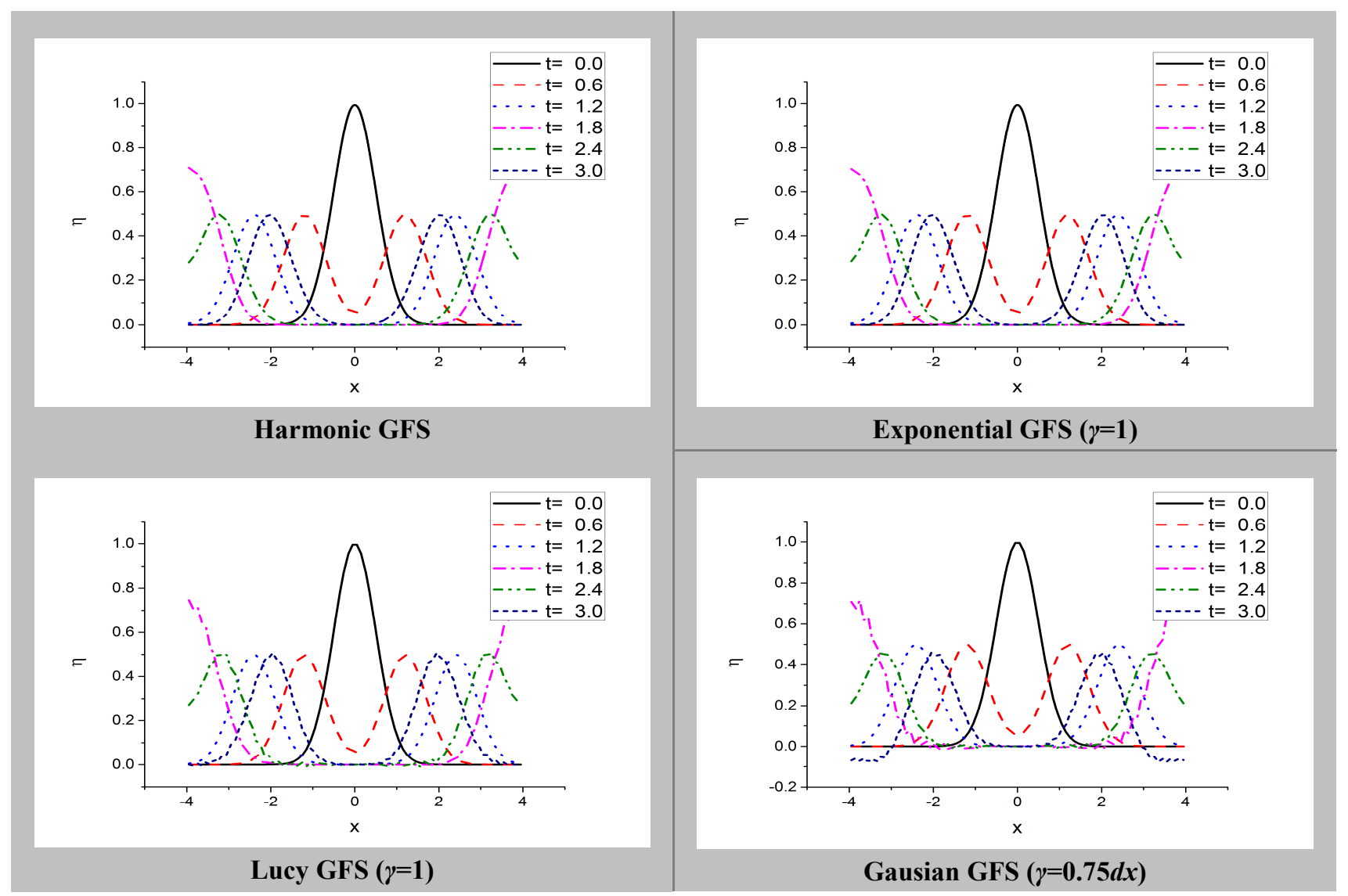

Figure 4. With reflection at boundaries

The discretization of Eqs. (35) and (36) is discussed in Ref. $[7,8]$. The solution of the Initial Boundary Value Problem (IBVP) is summarized as

$$
\begin{aligned}
& \text { From Eq. (35), } \eta(\mathbf{x}, t) \rightarrow \boldsymbol{\theta}(\mathbf{x}, t) ; \\
& \text { from (10), } \boldsymbol{\theta}(\mathbf{x}, t) \rightarrow \mathbf{q}(\mathbf{x}, t) ; \\
& \text { from (36), } \mathbf{q}(\mathbf{x}, t) \rightarrow \partial^{2} \eta(\mathbf{x}, t) / \partial t^{2} ; \\
& \text { from Eq. (28a), } \partial^{2} \eta(\mathbf{x}, t) / \partial t^{2} \rightarrow \partial \eta(\mathbf{x}, t+d t) / \partial t ; \\
& \text { from Eq. (28b), } \partial \eta(\mathbf{x}, t+d t) / \partial t \rightarrow \eta(\mathbf{x}, t+d t) ; \\
& \text { repeat. }
\end{aligned}
$$

For numerical calculations, same parameters given by Eq. (31) are used. The numerical results in Fig. 3 do not include reflection by walls, and the accurate results are obtained for all cases using Harmonic, exponential, Lucy and Gaussian GFSs. However, for cases involving reflection at walls as shown in Fig. 4, accurate numerical results are obtained only when Harmonic and Exponential GFSs are used. Small spurious oscillations are involved in numerical results using Lucy GFS. The numerical result using Gaussian GFS contains errors after reflection. This may correspond to our experience with respect to non-zero boundary values discussed in Ref. [8].

\section{Conclusions}

A set of integral representations is obtained using a fundamental solution of a differential-type boundary value problem [1-3]. If the boundary conditions are substituted into the set of the integral representations, a set of integral equations is obtained. The unknown variables of the boundary value problem can be determined by solving the set of integral equations derived from the set of integral representations.

Furthermore, we proposed a Generalized Integral Representation Method (GIRM) [4-8]. In GIRM, Generalized Fundamental Solution (GFS) is used. In the present paper, GIRM was applied to propagation of tidal wave. Unlike Integral Representation Method (IRM), we can use a variety of GFSs in GIRM. Four kinds of GFSs, namely Harmonic, Exponential, Lucy and Gaussian GFSs, were used. When the reflection of waves did not take place, all GFSs gave accurate numerical results. However, Lucy and Gaussian GFSs did not give reflected waves correctly enough. This may correspond to our experience with respect to non-zero boundary values discussed in Ref. [8].

In case of wave propagation discussed in the present paper, the second order time derivatives appear in the time evolution. An explicit time evolution is used successfully in the present paper. 


\section{Appendix A. Discretization of Eq. (27)}

The region $-L<x<+L$ is divided into $N$ intervals:

$$
\begin{gathered}
d x=2 L / N, \\
x_{i}=\xi_{i}=-L+(i+0.5) d x, i=0,1, \cdots, N-1,
\end{gathered}
$$

Equation (27) is discretized as follows:

$$
\begin{aligned}
& \sum_{j=0}^{N}\left[\frac{\partial^{2} \eta(\xi, t)}{\partial t^{2}}\right]_{\xi=\xi_{j}} \int_{x_{j}+d x / 2}^{x_{j}+d x / 2} \tilde{G}\left(\xi, x_{i}\right) d \xi \\
& =\sum_{j=0}^{N} \eta\left(\xi_{j}, t\right) \int_{x_{j}-d x / 2}^{x_{j}+d x / 2} \frac{\partial}{\partial \xi}\left(g h(\xi) \frac{\partial \tilde{G}\left(\xi, x_{i}\right)}{\partial \xi}\right) d \xi
\end{aligned}
$$

The integrals in Eq. (A2) can be evaluated numerically.

\section{References}

[1] Wu J.C., Thompson J.F., "Numerical solutions of time-dependent incompressible Navier-Stokes equations using an integro-differential formulations", Computers \& Fluids, (1973), 1, pp. 197-215.

[2] S. J. Uhlman, "An integral equation formulation of the equations of motion of an incompressible fluid", $N U W C-N P T$ Technical Report 10,086, 15 July, (1992).

[3] H. Isshik, S. Nagata, Y. Imai, "Solution of Viscous Flow around a Circular Cylinder by a New Integral Representation Method (NIRM)", AJET, 2, 2, (2014), pp. 60-82. file://C:/Users/1/Downloads/983-5001-1-PB\%20(1).pdf
[4] H. Isshik, S. Nagata, Y. Imai, "Solution of a diffusion problem in a non-homogeneous flow and diffusion field by the integral representation method (IRM)", Applied and Computational Mathematics, 3(1), (2014), pp. 15-26. $\mathrm{http}: / /$ article.sciencepublishinggroup.com/pdf/10.11648.j.acm. 20140301.13.pdf

[5] H. Isshiki, Theory and application of the generalized integral representation method (GIRM) in advection diffusion problem, Applied and Computational Mathematics, 3(4), (2014), pp. 137-149.

http://article.sciencepublishinggroup.com/pdf/10.11648.j.acm. 20140304.15.pdf

[6] H. Isshiki, T, Takiya and H. Niizato, Application of Generalized Integral representation (GIRM) Method to Fluid Dynamic Motion of Gas or Particles in Cosmic Space Driven by Gravitational Force, Applied and Computational Mathematics, Special Issue: Integral Representation Method and Its Generalization, (2015), under publicaion. $\mathrm{http} / /$ www.sciencepublishinggroup.com/journal/archive.aspx? journalid $=147 \&$ issueid $=-1$

[7] H. Isshiki, From Integral Representation Method (IRM) to Generalized Integral Representation Method (GIRM), Special Issue: Integral Representation Method and Its Generalization, (2015), under publicaion. http://www.sciencepublishinggroup.com/journal/archive.aspx? journalid=147\&issueid=-1

[8] H. Isshiki, Effects of Generalized Fundamental Solution (GFS) on Generalized Integral Representation Method (GIRM), Special Issue: Integral Representation Method and Its Generalization, (2015), under publicaion. http://www.sciencepublishinggroup.com/journal/archive.aspx? journalid=147\&issueid $=-1$ 